Article

\title{
Soft Pneumatic Bending Actuator with Integrated Carbon Nanotube Displacement Sensor
}

\author{
Tim Giffney, Mengying Xie, Aaron Yong, Andrew Wong, Philippe Mousset, Andrew McDaid and \\ Kean Aw * \\ Mechatronics Engineering, University of Auckland, 20 Symonds Street, Auckland 1010, New Zealand; \\ tim.giffney@auckland.ac.nz (T.G.); mxie008@aucklanduni.ac.nz (M.X.); ayon352@aucklanduni.ac.nz (A.Y.); \\ awon369@aucklanduni.ac.nz (A.W.); pmou407@aucklanduni.ac.nz (P.M.); \\ andrew.mcdaid@auckland.ac.nz(A.M.) \\ * Correspondence: k.aw@auckland.ac.nz, Tel.: +64-9-923-9767; Fax: +64-9-373-7479 \\ Academic Editor: Gursel Alici \\ Received: 31 October 2015; Accepted: 17 February 2016; Published: 24 February 2016
}

\begin{abstract}
The excellent compliance and large range of motion of soft actuators controlled by fluid pressure has lead to strong interest in applying devices of this type for biomimetic and human-robot interaction applications. However, in contrast to soft actuators fabricated from stretchable silicone materials, conventional technologies for position sensing are typically rigid or bulky and are not ideal for integration into soft robotic devices. Therefore, in order to facilitate the use of soft pneumatic actuators in applications where position sensing or closed loop control is required, a soft pneumatic bending actuator with an integrated carbon nanotube position sensor has been developed. The integrated carbon nanotube position sensor presented in this work is flexible and well suited to measuring the large displacements frequently encountered in soft robotics. The sensor is produced by a simple soft lithography process during the fabrication of the soft pneumatic actuator, with a greater than $30 \%$ resistance change between the relaxed state and the maximum displacement position. It is anticipated that integrated resistive position sensors using a similar design will be useful in a wide range of soft robotic systems.
\end{abstract}

Keywords: soft actuator; soft lithography; carbon nanotube; sensor

\section{Introduction}

In soft robotics, the rigid structures of traditional "hard" robots are replaced with innovative, compliant designs fabricated using flexible materials. Typical "hard" robots can perform prescribed tasks repetitively and with extreme precision. However, it is challenging for traditional robots to safely interact with delicate objects or operate in unstructured environments. In contrast, flexible materials and large strain deformations make soft robotics ideal for applications, such as human-robot interaction or squeezing between obstacles [1]. Examples of soft robots developed to date are grippers for fragile objects [2], biomedical robots, such as hand rehabilitation devices [3], and biomimetic systems, including crawling tetrapods [4] and manipulators inspired by octopus arms [5].

Realising the potential of soft robotics has required novel actuator designs. In a traditional robot, actuation occurs at joints connected by rigid members. In contrast, in soft robots, actuation is distributed throughout the flexible members of the system [1]. Actuation mechanisms for soft robots include dielectric elastomers [6], ionic polymer-metal composites $[7,8]$ and soft pneumatic actuators, such as fibre-reinforced actuators [9] or pneumatics networks (PneuNets) bending actuators [10]. PneuNets bending actuators use controlled inflation of moulded expandable chambers to achieve large bending displacements and are a flexible design well suited to varied soft robotics applications. 
Soft robotics presents special challenges for position sensing and control [11]. Traditional "hard" robots are rigid, and typical operating loads only cause small deflections of the robot structure. In hard robots, a small number of sensors at the joints of the robot can be used to measure the robot position, and excellent positioning accuracy may be achieved even with open loop control. In contrast, soft robots have much higher compliance, and even small applied forces can cause significant deformation. Additionally, this deformation is distributed across the entire flexible structure of the soft robot. As such, for accurate control of soft robots, position sensing is both more necessary and more difficult.

Conventional rigid sensors, such as encoders and metal or silicon strain gauges, are ill suited to soft robotics applications [11]. Instead, novel integrated sensors must be developed. In previous research, deformation sensing for soft robotics has been accomplished by techniques including optical measurement using light sources and light sensors mounted on the robot surface [12], capacitive sensing using conductive layers on either side of a stretchable dielectric [13] or resistive strain transducers exploiting the change in resistance that occurs when microfluidic channels containing liquid metals or conductive grease are deformed [14,15]. In order to avoid the need for external parts, a complex sensing circuit, or the fabrication of liquid-filled channels, an alternative design of an integrated sensor is desired.

Due to the mechanical and electrical properties of carbon nanotubes (CNT), electrically-conductive CNT networks on soft polymer substrates can be used to produce highly stretchable resistive strain sensors [16]. Additionally, carbon nanotube resistive strain sensors can be fabricated using low-cost soft lithography techniques [17]. In this work, a soft pneumatic bending actuator with an integrated carbon nanotube position sensor is demonstrated by integrating a carbon nanotube resistive large-strain sensor with a PneuNets-type soft pneumatic bending actuator. The carbon nanotube resistive sensor is easily integrated during the fabrication of the soft bending actuator by a simple soft lithography process and is able to measure the position of the soft bending actuator over a displacement range of $270^{\circ}$. In comparison to current state of the art sensors using liquid metals or conductive grease, this sensor has the advantages of easier integration in the fabrication process, a thin 0.3-mm thickness (avoiding stiffening of the actuator structure) and, due to the moulding process used for fabrication, is expected to be ideally suited for manufacturing sensors at multiple locations at one time.

\section{Concept}

The soft pneumatic bending actuator used in this work followed the PneuNets design [18] and was fabricated using Ecoflex ${ }^{\circledR}$ 00-30, an addition curing silicone material with Shore hardness 00-30 [19]. The integrated carbon nanotube resistive sensor used multi-walled carbon nanotubes (MWCNT) purchased from Shenzhen Nanotech Port Co. Ltd. (Shenzhen, China), with a diameter in the range of $10-30 \mathrm{~nm}$ and a length $>2 \mu \mathrm{m}$. The overall structure of the soft robotic actuator with integrated sensor is illustrated in Figure 1. The key dimensions of the fabricated device are listed in Table 1.

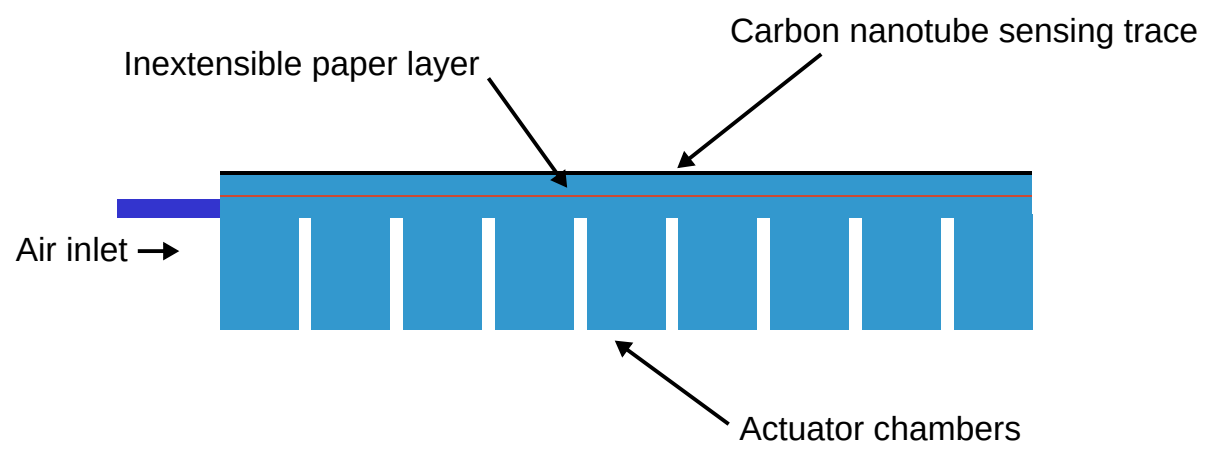

Figure 1. Simplified diagram of the soft robotic bending actuator with the integrated carbon nanotube position sensor. 
PneuNets bending actuators consist of a series of interconnected thin-walled air chambers moulded in silicone and bonded to a comparatively stiff upper layer [10]. The upper layer itself consists of a relatively inextensible paper sheet coated on each side with silicone [10]. When the air pressure inside the chambers is increased, the chambers inflate without significantly stretching the upper layer.

Table 1. Dimensions of the fabricated device.

\begin{tabular}{cc}
\hline Dimension & Value \\
\hline Overall length & $110 \mathrm{~mm}$ \\
Overall width & $17 \mathrm{~mm}$ \\
Number of chambers & 9 \\
Chamber height & $16 \mathrm{~mm}$ \\
Chamber facing wall thickness & $1.5 \mathrm{~mm}$ \\
MWCNT trace width & $0.6 \mathrm{~mm}$ \\
MWCNT trace thickness & $0.3 \mathrm{~mm}$ \\
MWCNT total trace length & $400 \mathrm{~mm}$ \\
\hline
\end{tabular}

In our device, the carbon nanotube position sensor is fabricated on the top surface of the upper layer. As the applied pressure is increased and the actuator deflects upwards, the carbon nanotube traces are compressed, leading to a decrease in resistance. It is expected that this design, in which the sensing element is compressed rather than stretched during actuation, will avoid issues with the cracking of the carbon nanotube composite that can occur under excessive tensile strain.

\section{Fabrication}

The soft pneumatic bending actuator and integrated carbon nanotube sensor were produced using a soft lithography process with the four moulds shown in Figure 2. Where possible, the moulds were produced using a laser-cut and laser-engraved acrylic sheet, with CNCmachining of the wax material used for the more complex geometry of the outer chamber mould.

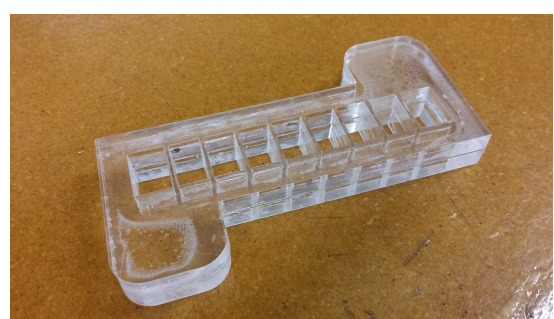

(a)

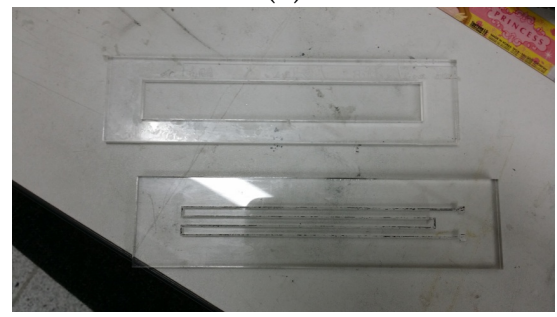

(c)

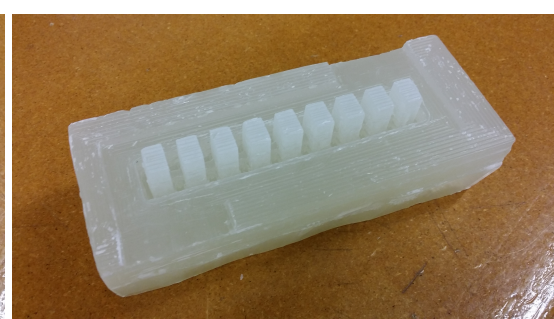

(b)

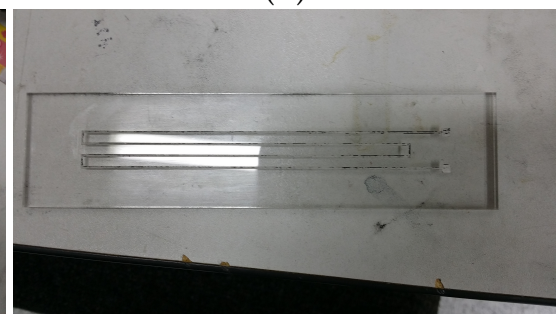

(d)

Figure 2. Moulds used for the fabrication process. (a) Outer chamber mould; (b) Inner chamber mould; (c) Upper layer mould; (d) Carbon nanotube mould.

First, the air chambers of the PneuNets actuator were fabricated using the outer and inner chamber moulds. Next, the carbon nanotube sensor and actuator upper layer were fabricated using the upper 
layer and carbon nanotube moulds. Finally, the assemblies were bonded together using more Ecoflex ${ }^{\circledR}$ as the adhesive.

\subsection{Chambers of the Soft Pneumatic Bending Actuator}

The initial step in the fabrication was to form the chambers of the soft pneumatic actuator itself. The procedure for fabricating the PneuNets soft actuator followed that presented by the Harvard Biodesign Lab Soft Robotics Toolkit [18]. In summary, Ecoflex ${ }^{\circledR}$ 00-30 Part A and Part B were mixed in a 1:1 ratio and cast in the assembled inner and outer chamber moulds shown in Figure 3. The assembly was left to cure for four hours at room temperature before removal from the mould.

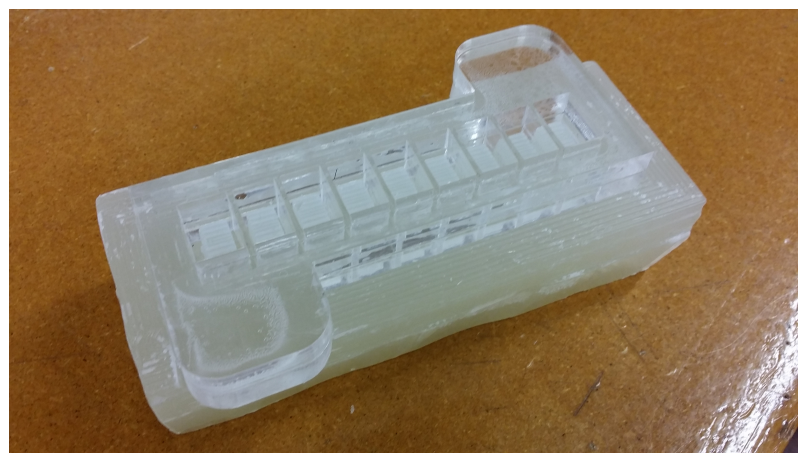

Figure 3. Outer and inner chamber moulds assembled for casting of actuator chambers.

\subsection{Carbon Nanotube Sensor}

The carbon nanotube resistive sensor was produced by a simple soft lithography process using the laser-engraved acrylic mould (shown in Figure 2d) and a 0.5\% dispersion of MWCNT in isopropanol (IPA) by weight.

First, the dispersion of MWCNT in IPA was prepared by the addition of $0.05 \mathrm{~g}$ MWCNT to $10 \mathrm{~g}$ of IPA, followed by sonication for $30 \mathrm{~min}$ in an Elmasonic ultrasound system to disperse the carbon nanotubes. An excess of the resulting MWCNT dispersion was then dispensed into the carbon nanotube mould, followed by spreading with a flat sheet to remove excess until there were no remaining gaps in the engraved track on the mould. The filled mould was then dried under vacuum to remove remaining IPA.

Finally, the Ecoflex ${ }^{\circledR}$ mixture was poured into the mould to form the upper layer of the soft pneumatic actuator and bonded to the MWCNT, as shown in Figure 4.

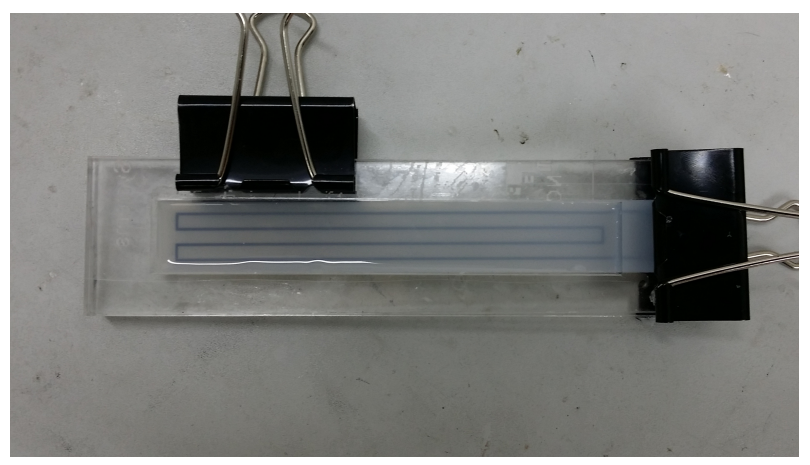

Figure 4. The uncured Ecoflex ${ }^{\circledR}$ mixture is poured into the assembled upper layer and CNT moulds to transfer the MWCNT large strain sensor to the Ecoflex ${ }^{\circledR}$ surface. 
The completed upper layer with the MWCNT sensor is shown in Figure 5. As shown in Figure 5, the MWCNT layer has become adhered to the silicone material due to the penetration of Ecoflex ${ }^{\circledR}$ into the MWCNT layer during curing.

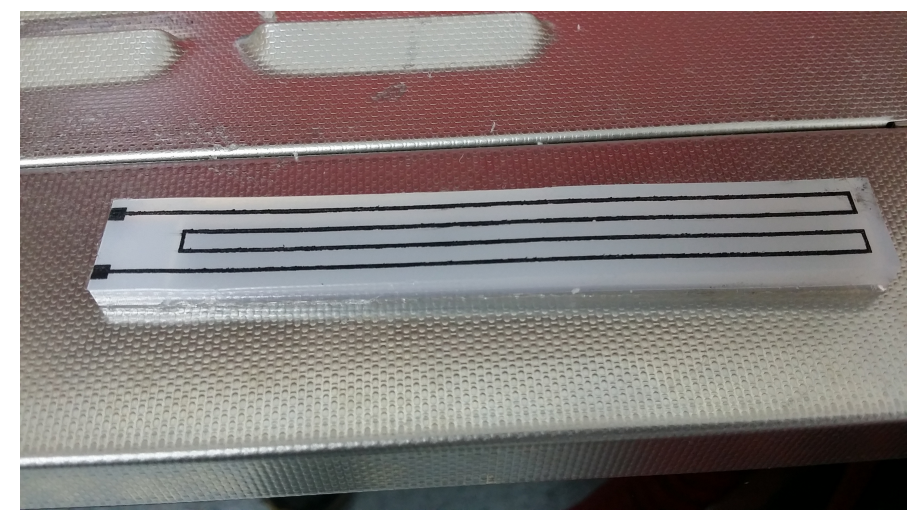

Figure 5. Completed upper layer with integrated CNT large strain sensor.

\subsection{Final Assembly}

In the final stage of the assembly process, the cured chamber assembly and upper layer were joined using additional Ecoflex ${ }^{\circledR}$ 00-30 mixture, which was spread on the top surface of the chamber assembly and the lower surface of the upper layer. To complete the fabrication process, after curing the final assembly for $4 \mathrm{~h}$ at room temperature, it was placed in an oven at $80^{\circ} \mathrm{C}$ for $2 \mathrm{~h}$ and $100{ }^{\circ} \mathrm{C}$ for $1 \mathrm{~h}$ to fully cure the Ecoflex ${ }^{\circledR}$. The completed soft pneumatic actuator with integrated MWCNT sensor is shown in Figure 6.

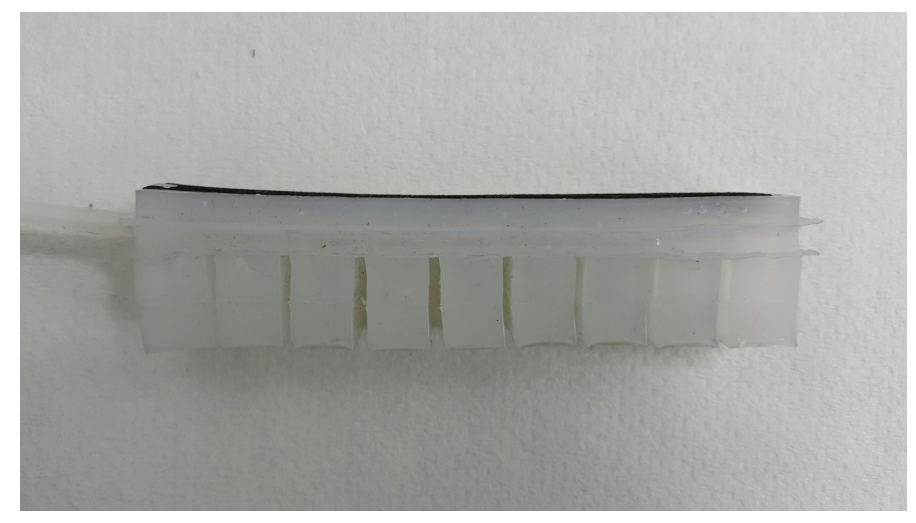

Figure 6. Completed soft pneumatic actuator with integrated MWCNT sensor.

\section{Characterization}

After fabrication, the performance of the bending actuator with the integrated carbon nanotube position sensor was characterized using an experimental system controlled from a computer to pressurize the actuator and measure bending angle and resistance.

An electro-pneumatic regulator valve (SMC ITV0030-3BS) was used to supply the actuator with a controllable air pressure between 0 and $500 \mathrm{kPa}$. At the same time, the angular position of the tip of the actuator was measured using a fixed camera and image processing software using the OpenCV library [20] to detect the rotation angle of a fiducial marker fixed to the end of the soft actuator. The resistance of the carbon nanotube large-strain sensor was measured using a Keithley 2602 source 
measure unit (SMU). In order to make a reliable connection to the carbon nanotube traces, the 3D printed mechanical clamp shown in Figure 7 was used to hold the electrodes in place.

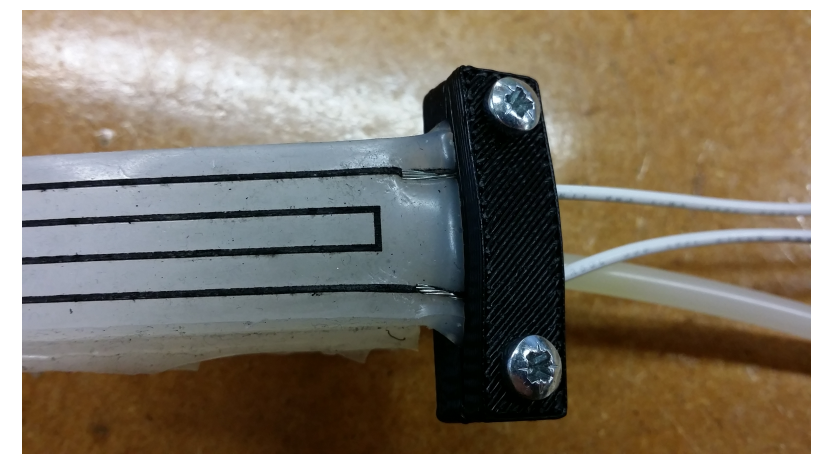

Figure 7. 3D-printed mechanical clamp used to ensure reliable connection to CNT traces.

\section{Results and Discussion}

The bending of the soft pneumatic actuator when the applied pressure is increased is shown in Figure 8. As shown in Figure 8, the actuator is capable of bending by more than 180 degrees. Due to the gap between adjacent actuator chambers in the relaxed state and the hyperelastic nature of the Ecoflex ${ }^{\circledR}$ material, the pneumatic pressure and the bending angle are not linearly related. As a result, very little actuator displacement occurs at low pressure, while at higher pressures, the angle of bending increases rapidly.

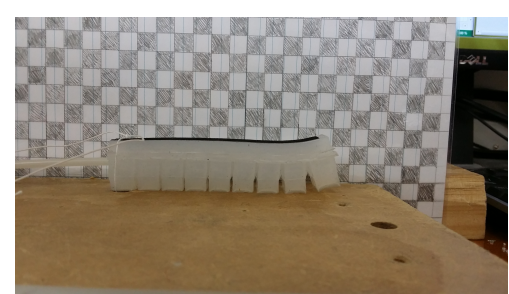

(a)

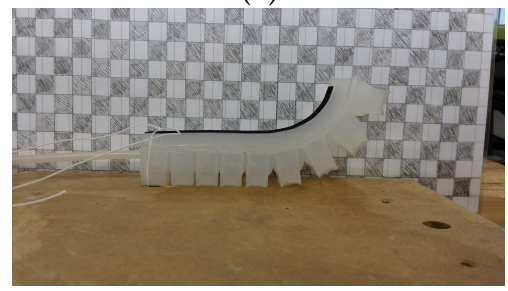

(d)

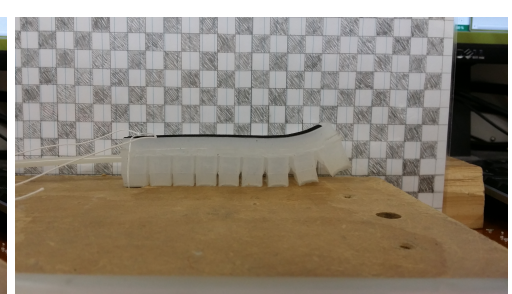

(b)

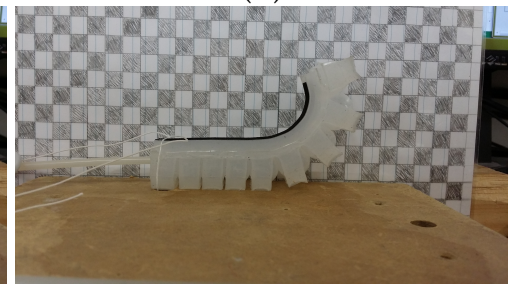

(e)

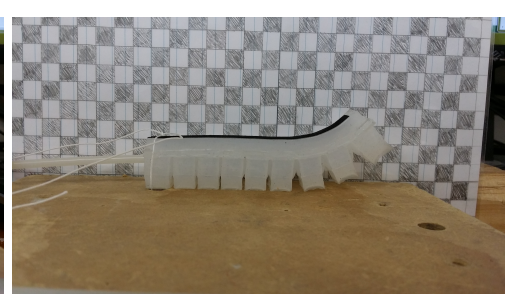

(c)

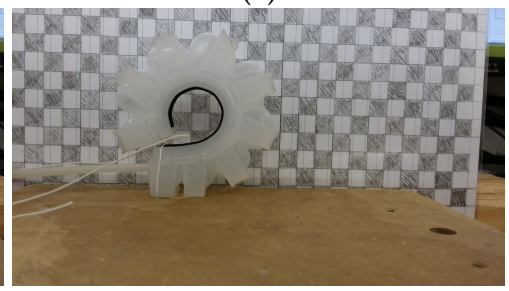

(f)

Figure 8. Images showing the soft actuator bending as the applied pressure is increased. (a) $6 \mathrm{kPa}$; (b) $7 \mathrm{kPa}$; (c) $8 \mathrm{kPa}$; (d) $9 \mathrm{kPa}$; (e) $10 \mathrm{kPa}$; (f) $11 \mathrm{kPa}$.

The change in resistance of the carbon nanotube sensor during bending is plotted against the bending angle in Figure 9. The change in resistance of a strained CNT layer depends on the combined effects of geometry change, tunnelling and physical disconnection effects, such as cracking [17]. From Figure 9, the relationship between bending angle and CNT strain sensor voltage is approximately linear $\left(R^{2}=0.996\right)$, as was also observed by other researchers at a similar strain level [21]. There is a substantial $38 \%$ decrease in sensor resistance between the relaxed and fully bent state, confirming that the integrated carbon nanotube large strain sensor is well suited to measuring the displacement of a soft pneumatic actuator. 
The hysteresis of the fabricated device is illustrated in Figure 10. From Figure 10, the largest difference between the measured resistance with increasing angle and decreasing angle was approximately $5 \%$ (occurring at $\theta=150^{\circ}$ ). Due to limitations of the experimental setup (where only the pressure in the actuator was controlled), it was challenging to control the rate of change of angle while deflating the actuator. For this reason, there are fewer data points in the curve for decreasing $\theta$ than the curve for increasing $\theta$. It is expected that some of the hysteresis may be explained by a different mechanical deflected shape during (faster) deflation than (slower) inflation.

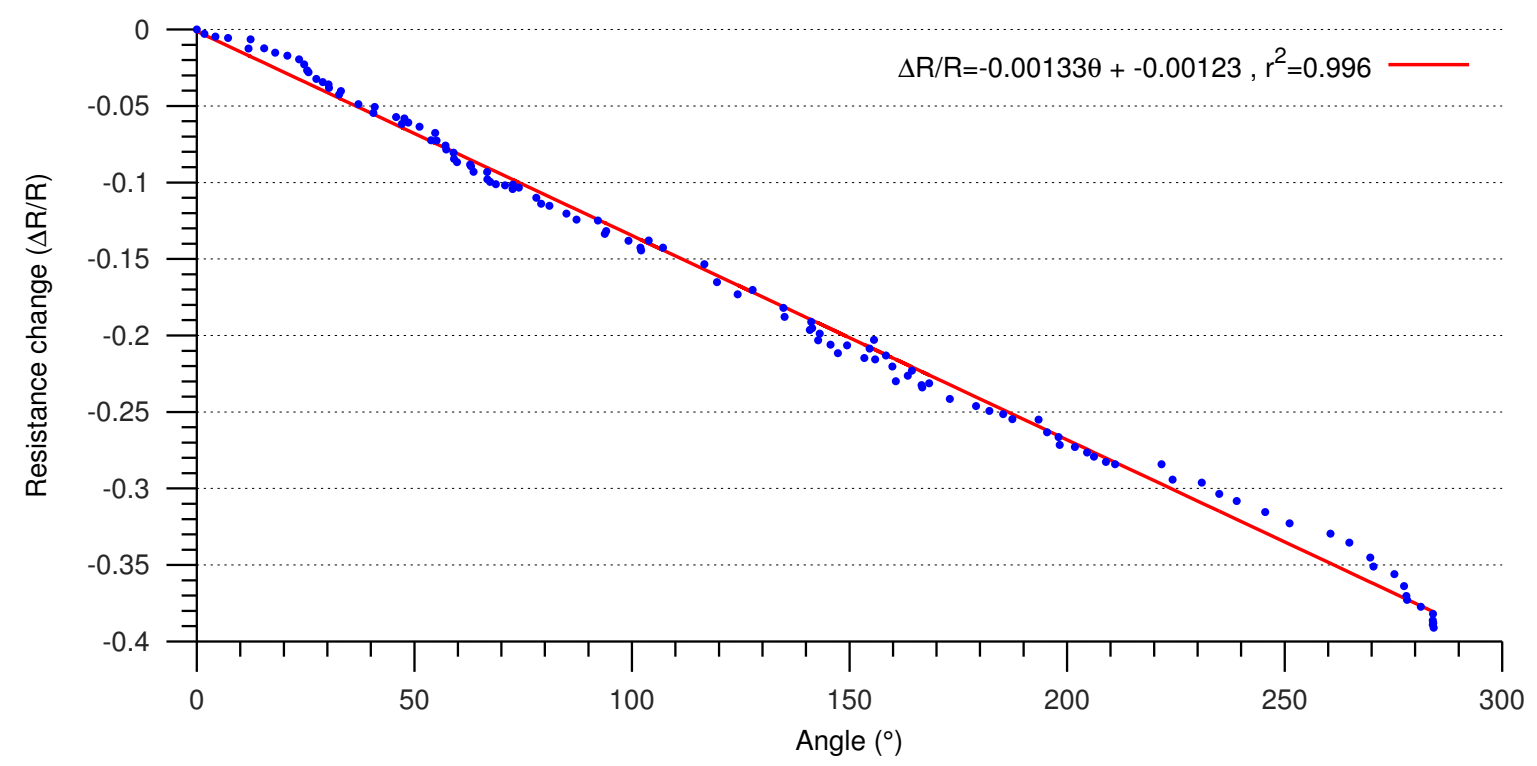

Figure 9. Relationship between the bending angle of the soft pneumatic actuator and the output voltage of the integrated carbon nanotube strain sensor.

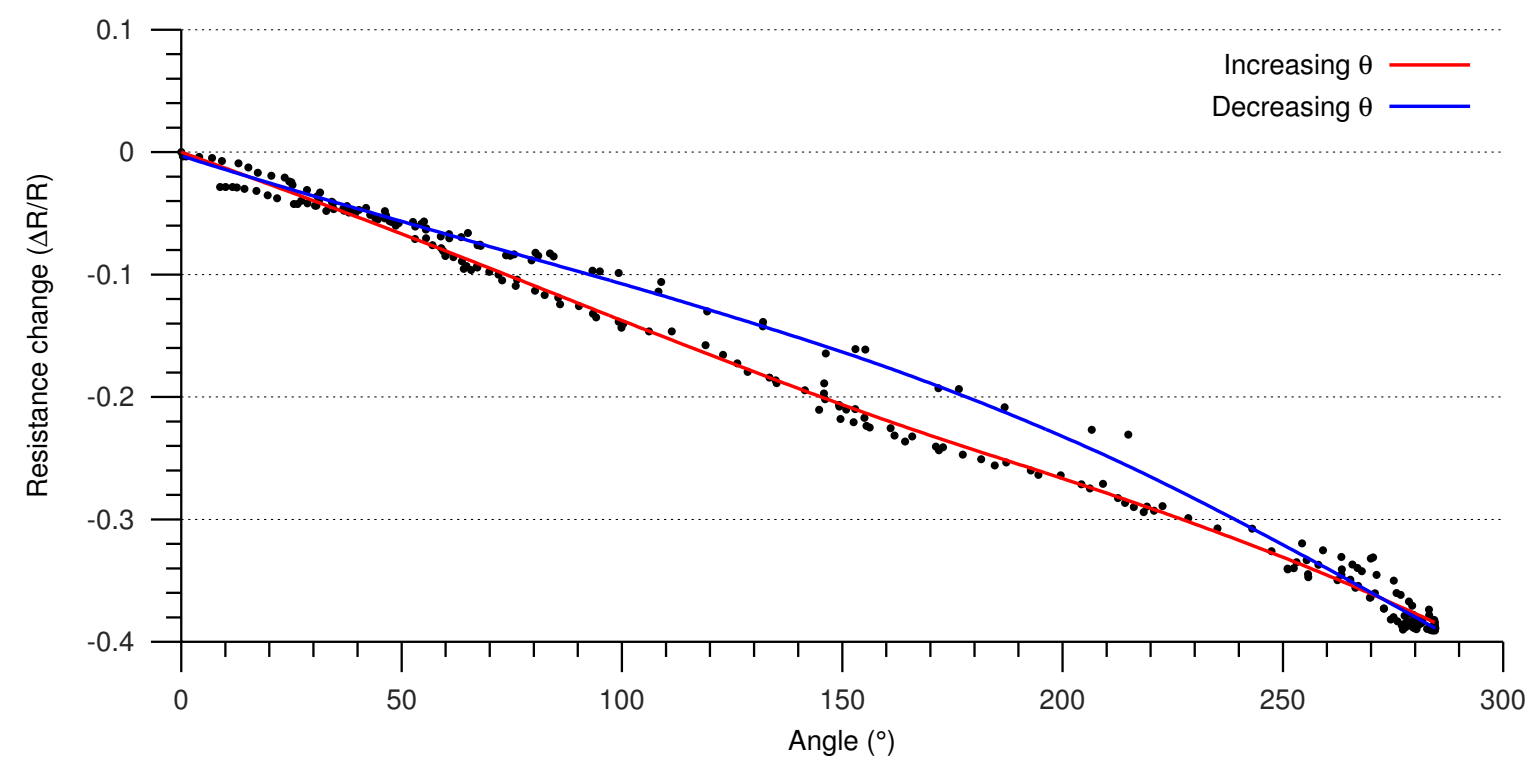

Figure 10. Hysteresis of the soft pneumatic actuator with the integrated carbon nanotube strain sensor. 


\section{Conclusions}

Historically, the flexible structure, large compliance and high displacements that make pneumatic soft robotic actuators attractive for biomimetic and human-robot interaction applications have also made the integration of suitable sensors for position measurement and control difficult. In this work, the position measurement of a soft pneumatic bending actuator has been realised using an integrated carbon nanotube large strain sensor. The carbon nanotube large strain sensor demonstrated in this work can effectively measure the angular displacement of the bending actuator, with sensor resistance varying by $38 \%$ between the relaxed state and the fully-deflected position and is easily integrated with the fabrication of the pneumatic actuator itself. It is expected that sensors of this type are also applicable to other soft robotics systems fabricated in cast silicone polymers.

Acknowledgements: The authors would like to acknowledge the assistance of Mingzhu Zhu for her detailed discussions and donation of the carbon nanotubes.

Author Contributions: Kean Aw and Andrew McDaid directed and guided this research. Mengying Xie and Tim Giffney designed the concept and process for the integrated carbon nanotube position sensor. Philippe Mousset, Andrew Wong and Aaron Yong developed testing software and fabricated and characterized the device.

Conflicts of Interest: The authors declare no conflict of interest.

\section{References}

1. Trivedi, D.; Rahn, C.; Kier, W.; Walker, I. Soft robotics: Biological inspiration, state of the art, and future research. Appl. Bionics Biomech. 2008, 5, 99-117.

2. Ilievski, F.; Mazzeo, A.D.; Shepherd, R.F.; Chen, X.; Whitesides, G.M. Soft Robotics for Chemists. Angew. Chem. Int. Ed. 2011, 50, 1890-1895.

3. Polygerinos, P.; Wang, Z.; Galloway, K.; Wood, R.; Walsh, C. Soft robotic glove for combined assistance and at-home rehabilitation. Robot. Auton. Syst. 2015, 73, 135-143.

4. Shepherd, R.; Ilievski, F.; Choi, W.; Morin, S.; Stokes, A.; Mazzeo, A.; Chen, X.; Wang, M.; Whitesides, G. Multigait soft robot. Proc. Natl. Acad. Sci. USA 2011, 108, 20400-20403.

5. Laschi, C.; Cianchetti, M.; Mazzolai, B.; Margheri, L.; Follador, M.; Dario, P. Soft Robot Arm Inspired by the Octopus. Adv. Robot. 2012, 26, 709-727.

6. Jung, K.; Koo, J.; Nam, J.D.; Lee, Y.; Choi, H. Artificial annelid robot driven by soft actuators. Bioinspir. Biomim. 2007, 2, S42-S49.

7. Yim, W.; Lee, J.; Kim, K. An artificial muscle actuator for biomimetic underwater propulsors. Bioinspir. Biomim. 2007, 2, S31-S41.

8. Aw, K.; McDaid, A. Bio-applications of ionic polymer metal composite transducers. Smart Mater. Struct. 2014, 23, doi:10.1088/0964-1726/23/7/074005 .

9. Galloway, K.; Polygerinos, P.; Walsh, C.; Wood, R. Mechanically programmable bend radius for fiber-reinforced soft actuators. In Proceedings of the 2013 16th International Conference on Advanced Robotics, ICAR 2013, Montevideo, Uruguay, 25-29 November 2013.

10. Mosadegh, B.; Polygerinos, P.; Keplinger, C.; Wennstedt, S.; Shepherd, R.; Gupta, U.; Shim, J.; Bertoldi, K.; Walsh, C.; Whitesides, G. Pneumatic networks for soft robotics that actuate rapidly. Adv. Funct. Mater. 2014, 24, 2163-2170.

11. Rus, D.; Tolley, M. Design, fabrication and control of soft robots. Nature 2015, 521, 467-475.

12. Dobrzynski, M.; Pericet-Camara, R.; Floreano, D. Contactless deflection sensor for soft robots. In Proceedings of the 2011 IEEE/RSJ International Conference on Intelligent Robots and Systems (IROS), San Francisco, CA, USA, 25-30 September 2011; pp. 1913-1918.

13. Girard, A.; Bigue, J.P.; O’Brien, B.; Gisby, T.; Anderson, I.; Plante, J.S. Soft Two-Degree-of-Freedom Dielectric Elastomer Position Sensor Exhibiting Linear Behavior. IEEE/ASME Trans. Mech. 2015, 20, 105-114.

14. Vogt, D.; Park, Y.L.; Wood, R. Design and characterization of a soft multi-axis force sensor using embedded microfluidic channels. IEEE Sens. J. 2013, 13, 4056-4064.

15. Muth, J.; Vogt, D.; Truby, R.; Menguc, Y.; Kolesky, D.; Wood, R.; Lewis, J. Embedded 3D printing of strain sensors within highly stretchable elastomers. Adv. Mater. 2014, 26, 6307-6312. 
16. Yamada, T.; Hayamizu, Y.; Yamamoto, Y.; Yomogida, Y.; Izadi-Najafabadi, A.; Futaba, D.; Hata, K. A stretchable carbon nanotube strain sensor for human-motion detection. Nat. Nanotechnol. 2011, 6, $296-301$.

17. Amjadi, M.; Yoon, Y.; Park, I. Ultra-stretchable and skin-mountable strain sensors using carbon nanotubes-Ecoflex nanocomposites. Nanotechnology 2015, 26, doi:10.1088/0957-4484/26/37/375501.

18. Polygerinos, P.; Mosadegh, B.; Campo, A. PneuNets Bending Actuators. Available online: http: //softroboticstoolkit.com/book/pneunets-bending-actuator (accessed on 28 October 2015).

19. Smooth-On, Inc. Available online: http://www.smooth-on.com/tb/files/ECOFLEX_SERIES_TB.pdf (accessed on 28 October 2015).

20. Bradski, G. The OpenCV Library; Dr. Dobbs J. 2000, 25; 120-125.

21. Amjadi, M.; Park, I. Carbon nanotubes-ecoflex nanocomposite for strain sensing with ultra-high stretchability. In Proceedings of 2015 28th IEEE International Conference on Micro Electro Mechanical Systems (MEMS), Estoril, Portugal, 18-22 January 2015, 2015, pp. 744-747.

(C) 2016 by the authors; licensee MDPI, Basel, Switzerland. This article is an open access article distributed under the terms and conditions of the Creative Commons by Attribution (CC-BY) license (http://creativecommons.org/licenses/by/4.0/). 